\title{
Fostering the Students' Capability of Solving Complex Engineering Problems through Systematic Design of Experimental Courses
}

\author{
Xiao-Wen ZHANG, Deng-Guang YU*, Jia WAN, Bo-Wen SUN, Hao FENG
}

School of Material Science and Engineering, University of Shanghai for Science and Technology, Shanghai 200093, China

*Email: ydg017@usst.edu.cn

${ }^{*}$ Corresponding author

Keywords: Higher education, Experiment courses, Complex engineering problem, Ability training, Electrospinning.

\begin{abstract}
In higher education, the college students should receive enough training to foster their capability of solving complex engineering problems in real world, which often occur during the material conversion processes from raw materials to target products in scientific laboratories. It is important that their experimental courses in their college studies are arranged in a systematic and complete manner. The scientific researches are often the epitomes of industrial productions. The advanced technologies happened in the laboratories can be exploited as the useful materials to train the capabilities of senior college students in resolving the engineering problems. Using the applications of electrospinning in creating solid dispersions of poorly water-soluble drugs as teaching materials, a series of experimental courses are systematically and completely arranged for the students in their course "Modern Material Analysis Methods". Through these training, the college students' abilities and experiences in resolving the complex engineering problems are expected to be enhanced.
\end{abstract}

\section{The Background for Systematic Design of Experiment Courses}

No matter in the industrial productions or in the experimental studies, a common procedure is that the activities start from the selections of raw materials, the determination of processing technologies, and end in the achievements of final products. Fig. 1 gives a schematic diagram of these activities. According to different situations, the raw materials can be natural materials, artificial materials, macroscopic materials and microscopic materials. As an nano era, more and more target products at microscopic scales are exploited as raw materials for deep processing. One famous example is the graphene oxides, which are frequently explored to prepare inorganic-organic composites to take advantages of their excellent physicochemical properties. However, the most important section during this procedure is the processing technology, which is often composed of a series of material processing methods. These methods include traditional techniques, advanced techniques, nanotechnologies, chemical synthesis approaches, and their combinations.

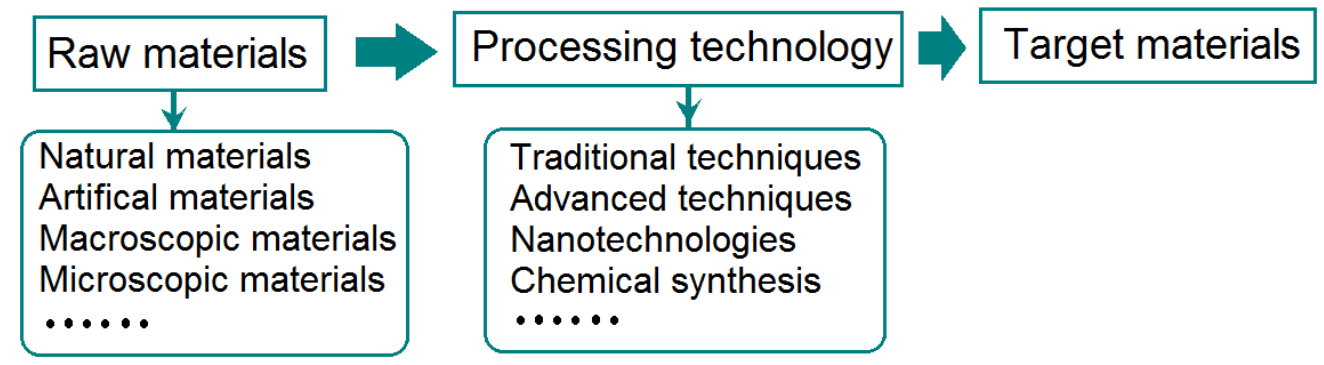

Figure 1. The complex engineering problems often occur during the material conversion processes from raw materials to target products.

In higher education, the college students in university of technology are required to grasp both the traditional industrial technologies and also the advanced scientific methods. But the most 
fundamental concept is that they should receive enough training to foster their capability of solving complex engineering problems in real world. Thus, it is very important that their experimental courses in their college studies should be systematic and complete.

\section{The Complex Engineering Problems Associated with the Advanced Technologies}

Because of the limited resources for educations, it is impossible that all the students are sent to the factories for receiving production practices for a long time period. However, there are many scientific research laboratories in all the universities. The scientific researches are often the epitomes of industrial productions. Thus, the advanced technologies happened in the laboratories can be exploited as the useful materials to train the capabilities of senior college students in resolving the engineering problems. The scientific researches can feedback the teaching in higher school not only in providing teaching materials, but also training them to think the experimental/industrial activities in a right manner.

Fig. 2 gives an example to how to organize a practice process. From a standpoint of developing novel materials, the first question and also the starting point is their functional applications. They can be materials for energy applications, environmental applications, biomedical applications, military applications, communication applications and any other applications. The second question is what type of raw materials can be utilized to create the desired products. The selection of origin materials needs experiences in the related fields. Then the key question is how to synthesize the desired products from the raw materials, i.e. the reasonable selection of technology. Today, the most popular techniques for material preparation are nanotechnologies, which can be divided into two categories, i.e. the top-down methods and bottom-up methods. Typical top-down methods are often a physical process without creation of new molecules such as grinding, high pressure homogenization, electrospinning and electrospraying.

In the higher education, the college students should be trained to grasp some of the up-to-date advanced technologies (particularly some of the nanotechnologies), to think over the whole material conversion processes, and to accumulate experiences in resolving the complex engineering problems associated with the advanced technologies. Thus, in future, it is should be easy for them to make use of these experience when they encounter the real complex engineering problems in their daily work. Here, an advanced technology, electrospinning [1-4], is utilized as a useful tool to explain the essences of complex engineering problems, to figure out how to design the experimental courses systematically in higher education for training the students' capability of solving complex problems.

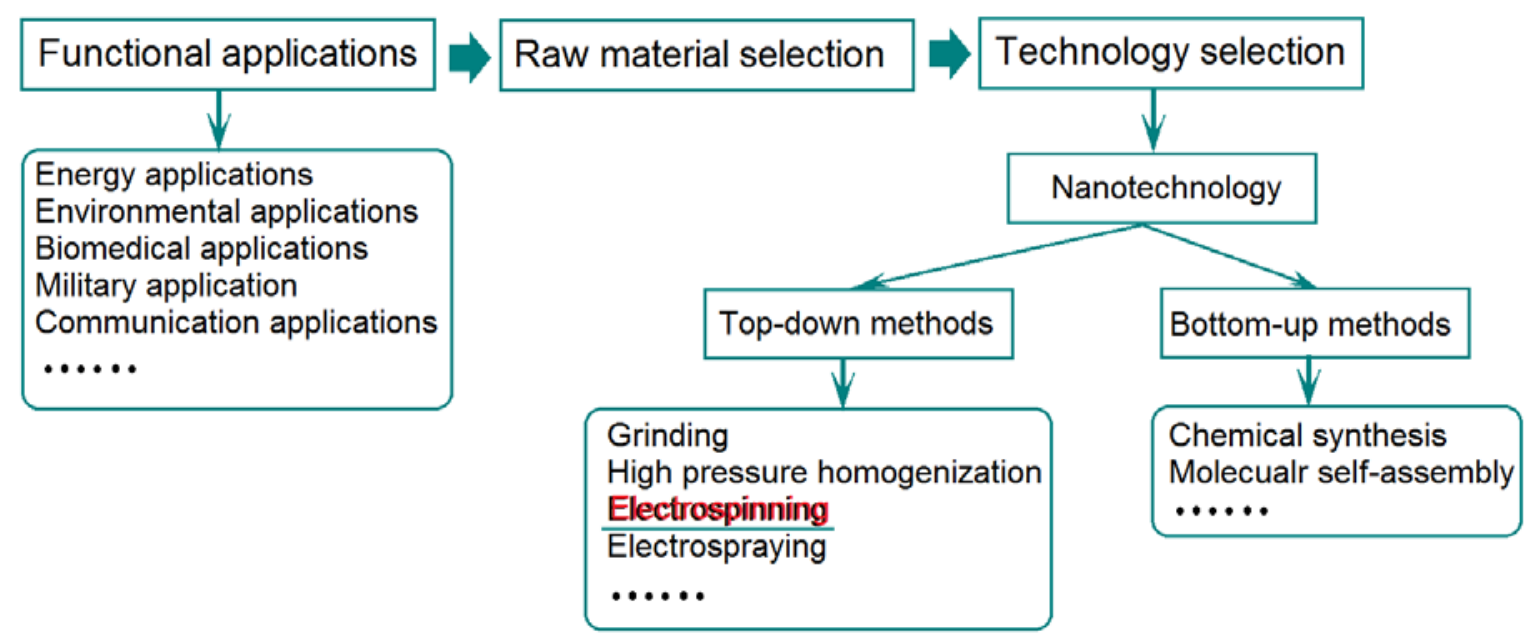

Figure 2. Advanced technologies can be utilized as the useful tools to explain the essences of complex engineering problems 


\section{The Line of Thinking about Solving a Complex Engineering Problem}

The dissolution and bioavailability of poorly water-soluble drugs are a long-standing question in pharmaceutics. Today the number of poorly soluble drugs has steadily increased. Estimates state that $40 \%$ of the drugs in the pipelines have solubility problems and about $60 \%$ of all drugs coming directly from synthesis are poorly soluble [5,6]. Many processes and technologies have been tried in order to provide more effective and versatile approaches to address them. Solid dispersion (SD) is one of the more promising strategies to improve the oral bioavailability of poor water soluble drugs. Electrospinning is a straightforward and versatile process by which polymer nanofibers with diameters ranging from a few nanometers to several micrometers can be produced using an electrostatically driven jet of polymer solution or polymer melt $[7,8]$. The usage of electrospun fibers formed as solid dispersion has gained widespread interest in pharmaceutics [9]. How to conceive and implement an electrospinning process to create nanofiber-based solid dispersions for poorly water-soluble drug comprises both a scientific question and also a complex engineering problem.

Shown in Figure 3 is a line of thinking about the electrospun solid dispersion. The key engineer problem is around the fabrication and characterization of final products. To solve this problem, a series of methods should be organized together to conduct a smooth preparation process and to determine the quality of as-prepared nanofiber-based solid dispersions. Correspondingly, both the preparation methods and the involved characterization methods should be taught to the college students in their experimental courses. And for training their abilities of solving complex engineering problems, these experimental courses should be arranged in a systematic manner.

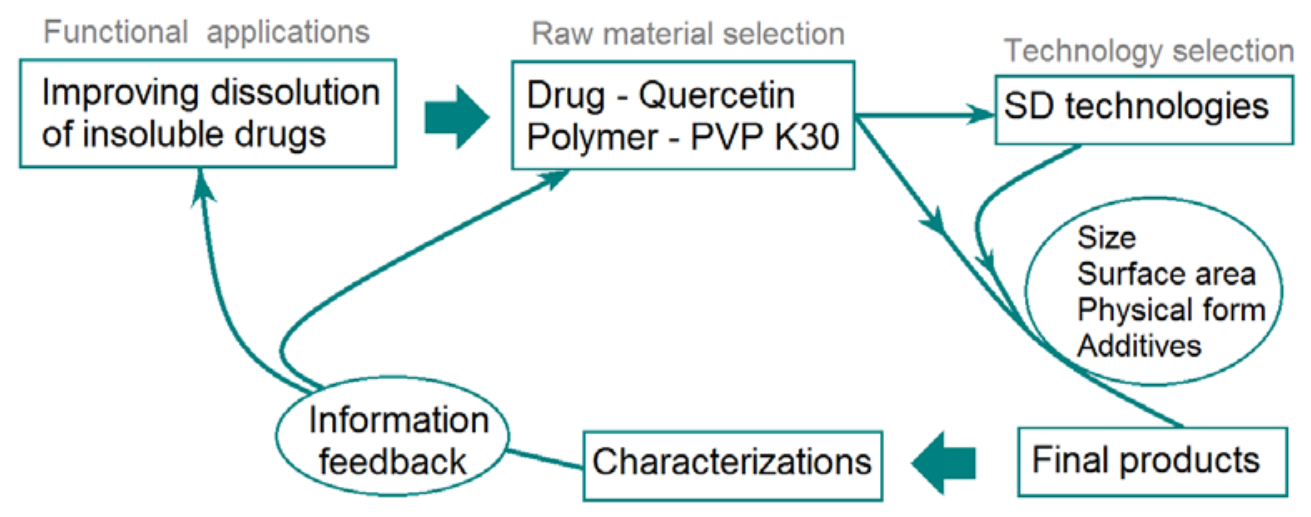

Figure 3. The line of thinking about solving a complex engineering problem - conceiving and implementing an electrospinning process to prepare solid dispersions containing poorly water-soluble drug.

\section{Systematic Design of Experimental Courses for Students’ Ability Training}

The capabilities of students in resolving complex engineering problems come from their comprehensive study, the application of their knowledge and systematic training. Shown in Figure 4, a series of analysis methods are systematically arranged for the students in their course "Modern Material Analysis Methods". The analysis methods include the protocols for carrying out the preparation of fiber-based solid dispersions using an electrospinning process, and the contents for analysizing and characterizing the fiber-based solid dispersions. The characterization processes can feedback useful information to improve the preparation processes, which involve a series of inter-related measures taken to optimize the engineering conditions. These contents should be excellent teaching materials for training the students' capabilities in resolving complex engineering problems.

For the preparation of nanofibers using an electrospinning process, the important contents include the preparation of working solution (using agitator and electronic balance), the measurements of their surface tensions (using surface tension measuring instrument), conductivities (using electrical 
conductivity meter), and rheological properties (using rheometer). These parameters have a close relationship with the electrospinnability of working solutions and should be considered in a systematic manner.

For the characterization of fiber-based solid dispersions, the typical contents include three aspects. Their morphology is able to be observed using scanning electron microscope (SEM), transmission electron microscope (TEM), optical microscope (OM), and atomic force microscope (AFM). The physical status of components and their compatibility can be determined using X-ray diffraction (XRD), differential scanning calorimetry (DSC) or thermal gravimetric analysis (TGA), Fourier transform infrared spectrometer (FTIR) and Roman spectroscopy. The functional performances of the prepared fiber-based solid dispersions can be detected using in vitro dissolution tests, ex vivo permeation tests, in vivo animal experiments, clinical experiments, and the drug concentration can be measured using UV-vis spectroscopy (UV) or High Performance Liquid Chromatography (HPLC). These experiments should be taught to the college students in systematic manner, and it is better to surround a novel material. Based on the systematic arrangement of experimental courses about material preparation and characterization, the college students' abilities and experiences in resolving the complex engineering problems are expected to be quickly improved.

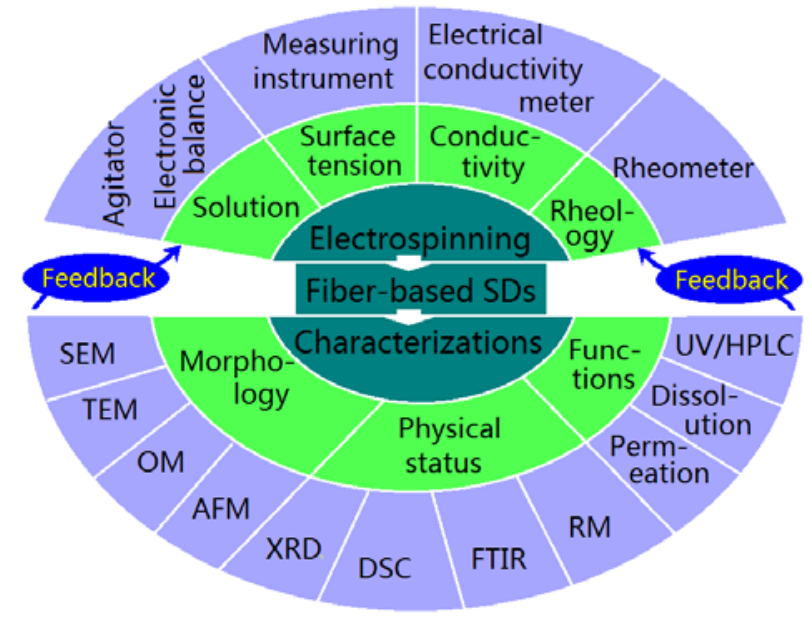

Figure 4. Systematic design of experimental courses for students’ ability training (SDs=solid dispersions).

\section{Summary}

The scientific researches are often the epitomes of industrial productions, which are similar from raw materials, through a series of processing technologies, and to the desired functional products. The advanced techniques utilized in the scientific laboratories can be exploited as the useful teaching materials to foster the capabilities of students in solving the complex engineering problems. Based on the applications of electrospinning in creating solid dispersions of poorly water-soluble drugs, a series of experimental methods are systematically and completely arranged for the senior students in their course "Modern Material Analysis Methods". Through these training, the college students' abilities and experiences in resolving the complex engineering problems are expected to be enhanced.

\section{Acknowledgments}

The financial supports from the following projects are appreciated: the National Natural Science Foundation of China (No. 51373101), 2016 key project on teachers teaching development in USST (CFTD16001Z), and the College Student Innovation Project of USST (Nos. XJ2016234 and SH2016176). 


\section{References}

[1] H.F. Wen, C. Yang, D.G. Yu, X.Y. Li and D.F. Zhang, Electrospun zein nanoribbons for treatment of lead-contained wastewater, Chem. Eng. J., 290 (2016), 263-272.

[2] D.G. Yu, C. Yang, M. Jin, G.R. Williams, H. Zou, X. Wang and S.W. Annie Bligh, Medicated Janus fibers fabricated using a Teflon-coated side-by-side spinneret, Colloid. Surf. B, 138 (2016) 110-116.

[3] B. Li, Z. Hua, Z. Liu, Study on preparing microcapsule in high-voltage electrostatic field. J. University of Shanghai for Science and Technology, 22 (2000) 189-193.

[4] D.G. Yu, X.Y. Li, X. Wang, J.H. Yang, S.W. Annie Bligh and G.R. Williams, Nanofibers fabricated using triaxial electrospinning as zero order drug delivery systems, ACS Appl. Mater. Interf., 7 (2015) 18891-18897.

[5] Y.H. WU, C. Yang, X.Y. Li, J.Y. Zhu, D.G. Yu, Medicated nanofibers fabricated using NaCl solutions as shell fluids in a modified coaxial electrospinning, J. Nanomater., 2016 (2016) Article ID 8970213, 12 pages.

[6] C. Yang, D.G. Yu, D. Pan, X.K. Liu, X. Wang, S.W. A. Bligh and G. R. Williams, Electrospun $\mathrm{pH}$-sensitive core-shell polymer nanocomposites fabricated using a tri-axial processes, Acta Biomater., 35 (2016) 77-86.

[7] F. Xu, Y. Xu and D.G. Yu, Zero-order controlled release nanofibers fabricated using coaxial electrospinning with polymer dilute solution as a sheath fluid, J. University of Shanghai for Science and Technology, 37 (2015) 165-168.

[8] D.G. Yu, K. White, N. Chatterton, Y. Li, L. Li and X. Wang, Structural lipid nanoparticles self-assembled from electrospun core-shell polymeric nanocomposites, RSC Adv., 5 (2015) 9462-9466.

[9] J. Yan, Y.H. Wu, D.G. Yu, G.R. Williams, S.M. Huang, W. Tao and J.Y. Sun, Electrospun acid-base pair solid dispersions of quercetin, RSC Adv., 4 (2014) 58265 - 58271. 